\title{
Mould-active compared with fluconazole prophylaxis to prevent invasive fungal diseases in cancer patients receiving chemotherapy or haematopoietic stem-cell transplantation: a systematic review and meta-analysis of randomised controlled trials
}

\author{
MC Ethier ${ }^{1,2}$, M Science ${ }^{2,3}$, J Beyene ${ }^{1,4}$, M Briel ${ }^{4,5}$, T Lehrnbecher ${ }^{6}$ and L Sung ${ }^{*, 1,7}$ \\ 'Program in Child Health Evaluative Sciences, The Hospital for Sick Children, Toronto M5G IX8, Ontario, Canada; ${ }^{2}$ Program in Health Research \\ Methodology, McMaster University, Hamilton L8S 4L8, Ontario, Canada; ${ }^{3}$ Division of Infectious Diseases, The Hospital for Sick Children, Toronto M5G \\ IX8, Ontario, Canada; ${ }^{4}$ Population Genomics Program, Department of Clinical Epidemiology and Biostatistics, McMaster University, Hamilton L8S 4L8, \\ Ontario, Canada; ${ }^{5}$ Basel Institute for Clinical Epidemiology and Biostatistics, Basel, Switzerland; ${ }^{6}$ Pediatric Hematology and Oncology, University of \\ Frankfurt, Frankfurt, Germany; 'Division of Haematology/Oncology, The Hospital for Sick Children, Toronto M5G IX8, Ontario, Canada
}

BACKGROUND: Objectives were to compare systemic mould-active vs fluconazole prophylaxis in cancer patients receiving chemotherapy or haematopoietic stem cell transplantation (HSCT).

METHODS: We searched OVID MEDLINE and the Cochrane Central Register of Controlled Trials (I948-August 20I I) and EMBASE (1980-August 20II). Randomised controlled trials of mould-active vs fluconazole prophylaxis in cancer or HSCT patients were included. Primary outcome was proven/probable invasive fungal infections (IFI). Analysis was completed by computing relative risks (RRs) using a random-effects model and Mantel-Haenszel method.

RESULTS: From 984 reviewed articles, 20 were included in this review. Mould-active compared with fluconazole prophylaxis significantly reduced the number of proven/probable IFI (RR $0.7 \mathrm{I}, 95 \% \mathrm{Cl} 0.52$ to $0.98 ; P=0.03$ ). Mould-active prophylaxis also decreased the risk of invasive aspergillosis (IA; RR 0.53, 95\% confidence interval (CI) $0.37-0.75 ; P=0.0004$ ) and IFI-related mortality (RR 0.67, 95\% Cl 0.47-0.96; $P=0.03$ ) but is also associated with an increased risk of adverse events (AEs) leading to antifungal discontinuation $(\mathrm{RR}$ ।.95, 95\% Cl I.24-3.07; $P=0.004)$. There was no decrease in overall mortality $(\mathrm{RR} \quad 1.0 ; 95 \% \mathrm{Cl} 0.88-1.13$; $P=0.96)$.

CONCLUSION: Mould-active compared with fluconazole prophylaxis significantly reduces proven/probable IFI, IA, and IFI-related mortality in cancer patients receiving chemotherapy or HSCT, but increases AE and does not affect overall mortality. (PROSPERO Registration: CRD420 I I I I74)

British Journal of Cancer (2012) I 06, 1626-1637. doi:I0.1038/bjc.2012.147 www.bjcancer.com

(C) 2012 Cancer Research UK

Keywords: antifungal; prophylaxis; fluconazole; infections

Cancer patients receiving intensive chemotherapy or undergoing haematopoietic stem cell transplantation (HSCT) are at an increased risk of invasive fungal infections (IFI); both yeasts and moulds contribute to IFI in these populations (Mahfouz and Anaissie, 2003). IFI are associated with considerable morbidity and mortality. Although invasive aspergillosis (IA) has decreased in recent years (Pagano et al, 2010), mortality remains unacceptably high. As a result, emphasis has been placed on prevention of IFI using prophylactic strategies.

Choices for systemic antifungal prophylaxis include fluconazole and agents with activity against moulds. Fluconazole is inexpensive and in general, well tolerated. However, it lacks activity against moulds, in particular against Aspergillus spp. In contrast, newer broad-spectrum azoles such as voriconazole and posaconazole,

*Correspondence: Dr L Sung; E-mail: lillian.sung@sickkids.ca Received 7 November 2011; revised 6 March 2012; accepted 22 March 2012 echinocandins such as caspofungin and micafungin, and amphotericin have coverage that extends to yeasts and moulds. However, each of these agents may have specific downsides including toxicity, potential for drug interactions and considerable costs.

Previous randomised trials have shown the benefits of fluconazole prophylaxis when compared with placebo in patients receiving chemotherapy and undergoing HSCT (Goodman et al, 1992; Slavin et al, 1995; Rotstein et al, 1999). However, individual trials comparing mould-active prophylaxis to fluconazole have yielded inconsistent results with most studies failing to show a reduction in proven or probable IFI (Wingard et al, 2010). Consequently, there are conflicting recommendations for antifungal prophylaxis from published guidelines (Cornely et al, 2009; Freifeld et al, 2011), which has led to variability in clinical practice (Lehrnbecher et al, 2009).

Although there are many randomised trials which assessed the efficacy of antifungal prophylaxis, most were underpowered to detect a significant difference in the incidence of proven or probable IFI or all-cause mortality. We hypothesised that 
including all available studies that compared mould-active $v s$ fluconazole prophylaxis would improve the ability to determine whether mould-active agents are associated with fewer proven or probable IFI and whether these agents are associated with a survival benefit. The primary objective of this review was to determine whether mould-active prophylaxis reduces the incidence of proven or probable IFI, when compared with fluconazole. The secondary objectives were to determine whether mould-active prophylactic strategies, when compared with fluconazole, are associated with a reduction in: (1) incidence of IA; (2) adverse events (AE) requiring discontinuation or modification of antifungal prophylaxis; (3) number of IFI- and IA-related deaths; and (4) all-cause mortality.

\section{METHODS}

The reporting of this meta-analysis follows the recommendations of the PRISMA (Preferred Reporting Items for Systematic Reviews and Meta-Analyses) statement (Moher et al, 2009). Methods of the analysis and inclusion criteria were specified in advance and registered in the PROSPERO registry of systematic reviews (Ethier, 2011).

\section{Eligibility criteria}

Randomised controlled trials comparing systemic mould-active to fluconazole prophylaxis were eligible. Any of the following mouldactive agents were included as long as they were administered systemically: amphotericin B (conventional and lipid formulations), caspofungin, micafungin, anidulafungin, posaconazole, itraconazole, voriconazole, or ketoconazole. Inclusion criteria were: (1) randomisation between systemic mould-active and fluconazole prophylaxis; and (2) patients of any age receiving chemotherapy for cancer or undergoing HSCT. We excluded (1) studies in which more than one systemic prophylactic anti-fungal agent was given in one of the study arms; (2) studies that did not report any of the primary or secondary outcomes; and (3) studies of pre-emptive or empiric therapy or anti-fungal treatment. There were no restrictions by language or by publication status.

\section{Information sources and search details}

We performed an electronic search of OVID MEDLINE (from 1948 to August 2011), EMBASE (from 1980 to August 2011), and The Cochrane Central Register of Controlled Trials (CENTRAL; until the third quarter of 2011). Searches were last updated 24 August 2011. We also reviewed the reference lists of relevant articles and reviews as well as trials registered on the ClinicalTrials.gov website. We searched for conference proceedings from 2005 to 2011 using the Web of Science (version 4.10) as well as abstracts presented within the last 2 years at annual meetings of the American Society of Hematology and American Society of Clinical Oncology.

We used the following search terms in both indexed and text word forms to search all databases: fluconazole, Aspergillus or mycoses, prevention or prophylaxis, neoplasm or SCT or neutropenia, with appropriate limits to identify randomised controlled trials (Appendix Table A1 for full search strategy). Two reviewers (MCE and MS) assessed the title and abstract of each reference identified by the search and applied the eligibility criteria. For potentially relevant articles, the full article was obtained and assessed by both authors independently. Final inclusion of studies in the meta-analysis was determined by agreement of both reviewers. If consensus could not be reached, disagreements were resolved by a third study author (LS). Agreement between reviewers was evaluated by using the kappa statistic.

\section{Outcomes}

The primary outcome was proven or probable IFI. IFI were re-classified using the revised EORTC/MSG criteria when sufficient data were available and authors used other definitions (De Pauw et al, 2008). When re-classification was not possible, the study was not included in the analysis of this outcome. A secondary outcome was IA that was defined as culture-proven Aspergillus or Aspergillus diagnosed by microscopic examination (De Pauw et al, 2008). The time period for IFI and IA observation was during the study period, which varied across studies. Other secondary outcomes were IFI- and IA-related mortality, all-cause mortality and adverse events leading to discontinuation or modification of study drug. The time period for observation of mortality was 3 months. We did not examine possible IFI as there was considerable inconsistency as to how this outcome was defined.

\section{Data collection process}

Two reviewers (MCE and MS) independently abstracted data from included trials using a standardised data collection form. Disagreements were resolved by discussion between the two reviewers; if no agreement could be reached, it was pre-specified that a third author (LS) would arbitrate. Corresponding authors were contacted to retrieve additional data if needed.

The following information was extracted: (1) study characteristics (recruitment period, number of subjects, follow-up period, country where study performed, whether study was multicenter, concurrent antibiotic prophylaxis, definitions for IFI, criteria for starting and stopping prophylaxis); (2) characteristics of trial participants (population, diagnosis, age, gender); (3) intervention and comparison (name of drug, dose, route, duration, frequency); and (4) outcomes.

\section{Risk of bias in individual studies}

To assess the risk of bias, included articles were examined by two reviewers (MCE and MS) for: (1) generation of sequence allocation; (2) allocation concealment; (3) blinding; (4) incomplete outcome data; and (5) intention-to-treat (ITT) analysis. Definitions/criteria of these items were derived from the Cochrane Handbook for Systematic Reviews of Interventions (Higgins and Green, 2009).

\section{Statistical analysis}

The meta-analysis was performed by computing relative risks (RRs) using a random-effects model as heterogeneity between trials was expected. We followed the ITT principle when we calculated summary RRs with $95 \%$ confidence intervals (CI). The Mantel-Haenszel method was chosen as the event rates were relatively low across all outcomes. We considered $P$-values $<0.05$ statistically significant. Synthesis was performed using Review Manager (Version 5.1, The Cochrane Collaboration, 2011, http:// ims.cochrane.org/revman/download). Meta-regression also was performed in addition to stratified analyses using SAS-PC software (version 9.2; SAS Institute, Cary, NC, USA).

Sub-group analyses defined a priori were performed to investigate the effects of age (children $v s$ adults), study population (HSCT vs chemotherapy), drug used in the experimental comparison group (amphotericin vs mould-active azoles $v s$ echinocandins), and dose of fluconazole $(\geqslant 400 \mathrm{mg}$ per day $v s$ $<400 \mathrm{mg}$ per day). We excluded studies that included both chemotherapy and HSCT patients from the study population subgroup analysis. We also examined subgroups by blinding and ITT analysis. Outcomes identified for sub-group analyses were proven/ probable IFI, IA, IFI-, and IA-related mortality, overall mortality and AEs requiring antifungal treatment discontinuation or modification. 
Concern has been raised about the effectiveness of itraconazole capsule to prevent IFD (Prentice et al, 2006) and at least two sets of guidelines have recommended against its use as prophylaxis (Walsh et al, 2008; Maertens et al, 2011). Consequently, we also conducted a sensitivity analysis excluding the two studies that used itraconazole capsules. There also has been concerns about whether doses of itraconazole solution $<400 \mathrm{mg}$ per day is effective (Glasmacher et al, 2003) and consequently, we conducted a second sensitivity analysis in which we deleted studies that used doses lower than this threshold amount.

Heterogeneity was initially inspected graphically (forest plot) and assessed statistically using the $I^{2}$ statistic and by performing a test for heterogeneity.

We assessed the possibility of publication bias by examining funnel plots for asymmetry (Sutton et al, 2000).

\section{RESULTS}

A total of 984 titles and abstracts were reviewed (Figure 1); 20 were retrieved for detailed evaluation (Bodey et al, 1994; Annaloro et al, 1995; Morgenstern et al, 1999a; Huijgens et al, 1999b; Timmers et al, 2000b; Wolff et al, 2000b; Koh et al, 2002a; Glasmacher et al, 2003; Winston et al, 2003; Marr et al, 2004; van Burik et al, 2004; Choi et al, 2005; Oren et al, 2006b; Cornely et al, 2007; Ito et al, 2007b; Ullmann et al, 2007; Hiramatsu et al, 2008b; Sawada et al, 2009a; Ota et al, 2010; Wingard et al, 2010) and all 20 (19 full-text articles and one conference abstract (Ota et al, 2010)) satisfied eligibility criteria and were included in the final meta-analysis. The kappa statistic for study inclusion was 1.0, reflecting perfect agreement.

Demographics of the 20 included studies are presented in Table 1. A total of 5725 patients were included in this review with ages ranging from 0.6 to 82 years. Trials were performed in Asia $(n=7)$, Europe $(n=5)$, North America $(n=5)$, and internationally $(n=3)$. Half of the studies were multi-centred (10 out of 20 , $50.0 \%)$. The patient populations were HSCT $(n=14)$ and chemotherapy $(n=6)$. Children were included in four trials but only one trial comprised of children only. Antibiotic prophylaxis was recommended in 8 out of $20(40 \%)$ of trials. Study regimens included amphotericin B formulations $(n=4)$, micafungin $(n=3)$, posaconazole $(n=2)$, voriconazole $(n=1)$, and itraconazole $(n=10)$. All studies of echinocandins consisted of micafungin. Fungal prophylaxis was started either with the initiation of chemotherapy $(n=18)$ or at the onset of neutropenia $(n=1$; Sawada et al, 2009a), and was not available for one study (Choi et al, 2005). Routine galatomannan testing was performed in two

$\rightarrow$

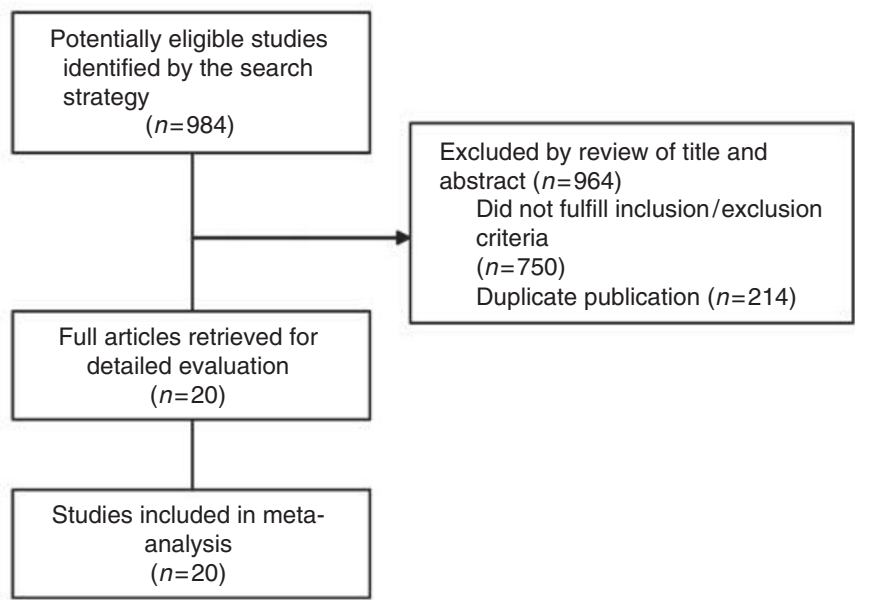

Figure I Flow diagram of trial identification and selection. trials and serum beta-D-glucan testing in one trial (Ito et al, 2007a; Ullmann et al, 2007; Wingard et al, 2010).

Risk of bias assessment is presented in Table 2. The majority of studies did not provide adequate information on sequence allocation and allocation concealment. Only 4 out of $20(20 \%)$ of the studies were blinded and 6 out of $20(30 \%)$ performed an ITT analysis.

The analysis of the primary outcome, which was proven or probable IFI, encompassed 2385 (mould-active group) and 2417 (fluconazole group) patients, in 18 studies. When data from all 18 studies that reported on our primary outcome were pooled, mould-active compared with fluconazole prophylaxis significantly reduced the risk of IFI (RR $0.71,95 \%$ CI $0.52-0.98 ; P=0.03$ ), with moderate heterogeneity $\left(I^{2}=33 \%, P=0.11\right)$ as illustrated in Table 3 and Figure 2.

Mould-active prophylaxis, when compared with fluconazole prophylaxis, decreased the risk of IA (RR 0.53 , 95\% CI $0.37-0.75$ ) and IFI-related mortality (RR $0.67,95 \%$ CI $0.47-0.96$ ). However, mould-active prophylaxis was significantly associated with more adverse events leading to antifungal prophylaxis discontinuation or modification when compared with fluconazole prophylaxis (RR $1.95,95 \%$ CI 1.24-3.07). Importantly, mould-active prophylaxis did not significantly influence overall mortality (RR 1.0, 95\% CI 0.88-1.13). Funnel plots were reviewed for each of the study outcomes. No apparent asymmetry was seen by visual assessment (data not shown).

The results from the subgroup analyses for 4 of our 5 prespecified outcomes are presented in Table 4 and Appendix Table A3. Subgroup analysis by age was not possible as only one study included children only. There was no evidence for a difference in the effect of mould-active $v s$ fluconazole prophylaxis for any of the outcomes. However, the beneficial effect of mouldactive prophylaxis appeared qualitatively greater in studies of other azoles and echinocandins in comparison with amphotericin B formulations. There was no evidence that the effect of mouldactive prophylaxis differed by blinding status or application of the ITT principle (Appendix Table A2). The results from the metaregression are presented in Appendix Table A4 and are consistent with the results from the sub-group analysis.

Appendix Table A5 illustrates the sensitivity analyses that removed the two studies of itraconazole capsule prophylaxis and the three studies that used oral itraconazole solution doses $<400 \mathrm{mg}$ per day. The removal of these studies did not impact the results, with the exception of proven or probably IFI which was no longer significant after removing the three studies that used oral itraconazole solution doses $<400 \mathrm{mg}$ per day.

\section{DISCUSSION}

We found that in patients with cancer receiving chemotherapy or HSCT, mould-active prophylaxis when compared with fluconazole prophylaxis was associated with a clinically relevant reduction in proven or probable IFI, IA and IFI-related mortality. However, mould-active prophylaxis was also associated with a significantly increased risk of adverse events requiring discontinuation or modification of therapy and did not affect overall mortality.

These results are in keeping with those from a previous review comparing mould-active to fluconazole prophylaxis that was conducted as a sub-group analysis of a large review; this review included studies published up to 2007 (Robenshtok et al, 2007). Similar to our study, that review found that mould-active prophylaxis significantly reduced documented IFI, IA, and IFIrelated mortality, and did not impact on all-cause mortality. Our results provide important new information since six new trials comparing fluconazole to systemic mould-active prophylaxis were added (Choi et al, 2005; Ito et al, 2007a; Hiramatsu et al, 2008a; Sawada et al, 2009b; Ota et al, 2010; Wingard et al, 2010), which 


\begin{tabular}{|c|c|c|c|c|c|c|c|c|}
\hline Study author & Pub year & $\begin{array}{l}\text { Multi- } \\
\text { centre }\end{array}$ & $\mathbf{N}$ & Population & Mould-active dose & Fluconazole dose & Prophylaxis end & $\begin{array}{c}\text { Surro- } \\
\text { gate } \\
\text { testing }\end{array}$ \\
\hline Bodey et al, 1994 & 1994 & No & 77 & Hem malignancy & $\begin{array}{l}\text { CAB } 0.17 \mathrm{mg} \mathrm{kg}^{-1} \text { per dose } \\
\text { IV TID }\end{array}$ & $\begin{array}{l}400 \text { mg per dose PO/IV } \\
\text { OD }\end{array}$ & $\begin{array}{l}\text { ANC> }>\left.1000 \mu\right|^{-1} \text { or } 8 \\
\text { weeks }\end{array}$ & No \\
\hline $\begin{array}{l}\text { Annaloro et al, } \\
1995\end{array}$ & 1995 & No & 59 & HSCT (auto, allo) & $\begin{array}{l}\text { Itraconazole } 400 \mathrm{mg} \text { per dose } \\
\text { PO OD }\end{array}$ & $300 \mathrm{mg}$ per dose PO OD & Neutropenia resolution & No \\
\hline $\begin{array}{l}\text { Huijgens et al, } \\
1999 \text { a }\end{array}$ & 1999 & No & 202 & $\begin{array}{l}\text { Hem malignancy, HSCT } \\
\text { (auto) }\end{array}$ & $\begin{array}{l}\text { Itraconazole } 100 \mathrm{mg} \text { per dose } \\
\text { PO BID }\end{array}$ & $50 \mathrm{mg}$ per dose $\mathrm{PO}$ BID & ANC $>\left.500 \mu\right|^{-1}$ & No \\
\hline $\begin{array}{l}\text { Morgenstern } \\
\text { et al, I } 1999 \mathrm{~b}\end{array}$ & 1999 & Yes & 581 & $\begin{array}{l}\text { Hem malignancy, HSCT } \\
\text { (auto, allo) }\end{array}$ & $\begin{array}{l}\text { Itraconazole } 2.5 \mathrm{mg} \mathrm{kg}^{-1} \text { per } \\
\text { dose PO BID }\end{array}$ & $100 \mathrm{mg}$ per dose PO OD & $\begin{array}{l}\text { ANC }>\left.1000 \mu\right|^{-1} \times 7 \\
\text { days }\end{array}$ & No \\
\hline $\begin{array}{l}\text { Timmers et al, } \\
2000 a\end{array}$ & 2000 & No & 24 & $\begin{array}{l}\text { Hem malignancy, HSCT } \\
\text { (auto, allo) }\end{array}$ & $\begin{array}{l}\text { Amphotericin B colloidal } \\
\text { dispersion } 2 \mathrm{mg} \mathrm{kg}^{-1} \text { per dose } \\
\text { IV OD }\end{array}$ & $200 \mathrm{mg}$ per dose $\mathrm{PO} O \mathrm{OD}$ & ANC $>500 \mu 1^{-1}$ & No \\
\hline $\begin{array}{l}\text { Wolff et al, } \\
2000 a\end{array}$ & 2000 & Yes & 355 & HSCT (auto, allo) & $\begin{array}{l}\text { CAB } 0.2 \mathrm{mg} \mathrm{kg}^{-1} \text { per dose IV } \\
\text { OD }\end{array}$ & $\begin{array}{l}400 \text { mg per dose PO/IV } \\
\text { OD }\end{array}$ & ANC $>500 \mu 1^{-1}$ & No \\
\hline Koh et al, 2002b & 2002 & No & 186 & HSCT (auto, allo) & $\begin{array}{l}\text { CAB } 0.2 \mathrm{mg} \mathrm{kg}^{-1} \text { per dose IV } \\
\text { OD }\end{array}$ & $200 \mathrm{mg}$ per dose $\mathrm{PO} O \mathrm{OD}$ & $\begin{array}{l}\text { ANC }>500 \mu 1^{-1} \times 3 \\
\text { days }\end{array}$ & No \\
\hline $\begin{array}{l}\text { Winston et al, } \\
2003\end{array}$ & 2003 & Yes & 138 & HSCT (allo) & $\begin{array}{l}\text { Itraconazole } 200 \mathrm{mg} \text { per dose } \\
\text { IV BID } \times 4 \text { then } 200 \mathrm{mg} \text { per } \\
\text { dose IV OD } \times 12 \text { then } 200 \mathrm{mg} \\
\text { per dose PO BID until } \\
D+100\end{array}$ & $\begin{array}{l}400 \mathrm{mg} \text { per dose IV } \\
\mathrm{OD} \times 14 \text { then } 400 \mathrm{mg} \\
\text { per dose PO OD until } \\
D+100\end{array}$ & $D+100$ & No \\
\hline Marr et al, 2004 & 2004 & No & 299 & HSCT (allo) & $\begin{array}{l}\text { Itraconazole } 2.5 \mathrm{mg} \mathrm{kg}^{-1} \text { per } \\
\text { dose PO TID or } 200 \mathrm{mg} \text { per } \\
\text { dose IV OD }\end{array}$ & $\begin{array}{l}400 \text { mg per dose PO/IV } \\
\text { OD }\end{array}$ & $D+120-180$ days & No \\
\hline $\begin{array}{l}\text { van Burik et al, } \\
2004\end{array}$ & 2004 & Yes & 882 & HSCT (auto, allo) & $\begin{array}{l}\text { Micafungin } 50 \mathrm{mg} \text { per dose IV } \\
\text { OD }\end{array}$ & $400 \mathrm{mg}$ per dose IV OD & $\begin{array}{l}\text { ANC } \geqslant 500 \mu 1^{-1} \times 5 \\
\text { days or } D+42\end{array}$ & No \\
\hline Choi et al, 2005 & 2005 & No & 78 & HSCT (allo) & $\begin{array}{l}\text { Itraconazole } 200 \mathrm{mg} \text { per dose } \\
\text { PO OD }\end{array}$ & $200 \mathrm{mg}$ per dose PO OD & NS & No \\
\hline $\begin{array}{l}\text { Glasmacher et al, } \\
2006\end{array}$ & 2006 & Yes & 494 & Hem malignancy & $\begin{array}{l}\text { Itraconazole } 5 \mathrm{mg} \mathrm{kg}^{-1} \text { per } \\
\text { dose PO BID }\end{array}$ & $\begin{array}{l}400 \mathrm{mg} \text { per dose } \mathrm{PO} / \mathrm{IV} \\
\text { OD }\end{array}$ & $\begin{array}{l}\text { ANC> }>\left.1000 \mu\right|^{-1} \text { or } 8 \\
\text { weeks }\end{array}$ & No \\
\hline Oren et al, 2006a & 2006 & No & 195 & $\begin{array}{l}\text { Hem malignancy, HSCT } \\
\text { (auto, allo) }\end{array}$ & $\begin{array}{l}\text { Itraconazole } 200 \mathrm{mg} \text { per dose } \\
\mathrm{PO} / \mathrm{IV} \text { BID }\end{array}$ & $\begin{array}{l}400 \mathrm{mg} \text { per dose } \mathrm{PO} / \mathrm{IV} \\
\mathrm{OD}\end{array}$ & $\begin{array}{l}\text { Neutropenia resolution or } \\
8 \text { weeks }\end{array}$ & No \\
\hline $\begin{array}{l}\text { Cornely et al, } \\
2007\end{array}$ & 2007 & Yes & 544 & Hem malignancy & $\begin{array}{l}\text { Posaconazole } 200 \text { mg per } \\
\text { dose PO/IV BID }\end{array}$ & $\begin{array}{l}400 \text { mg per dose PO/IV } \\
\text { OD }\end{array}$ & $\begin{array}{l}\text { Neutropenia resolution or } \\
12 \text { weeks }\end{array}$ & No \\
\hline Ito et al, 2007a & 2007 & Yes & 209 & Hem malignancy & $\begin{array}{l}\text { Itraconazole } 200 \mathrm{mg} \text { per dose } \\
\text { PO OD }\end{array}$ & $200 \mathrm{mg}$ per dose $\mathrm{PO} O \mathrm{OD}$ & $\begin{array}{l}\text { ANC }>\left.1000 \mu\right|^{-1} \text { or } \\
\text { leukocytes } \geqslant\left. 2 \mu\right|^{-1}\end{array}$ & Yes \\
\hline $\begin{array}{l}\text { Ullmann et al, } \\
2007\end{array}$ & 2007 & Yes & 600 & GVHD & $\begin{array}{l}\text { Posaconazole } 200 \mathrm{mg} \text { per } \\
\text { dose PO TID }\end{array}$ & $400 \mathrm{mg}$ per dose PO OD & 112 days & Yes \\
\hline $\begin{array}{l}\text { Hiramatsu et al, } \\
\text { 2008a }\end{array}$ & 2008 & No & 100 & HSCT (auto, allo) & $\begin{array}{l}\text { Micafungin } 150 \mathrm{mg} \text { per dose IV } \\
\text { OD }\end{array}$ & $400 \mathrm{mg}$ per dose IV OD & $\begin{array}{l}\text { ANC }>500 \mu 1^{-1} \times 5 \text { or } \\
D+42\end{array}$ & No \\
\hline $\begin{array}{l}\text { Sawada et al, } \\
2009 b\end{array}$ & 2009 & Yes & 107 & $\begin{array}{l}\text { Hem malignancy, HSCT } \\
\text { (allo/auto) }\end{array}$ & $\begin{array}{l}\text { Micafungin } 2 \mathrm{mg} \mathrm{kd}^{-1} \text { per } \\
\text { dose IV OD }\end{array}$ & $\begin{array}{l}10 \mathrm{mg} \mathrm{kg}^{-1} \text { per dose } \mathrm{IV} \\
\mathrm{OD}\end{array}$ & $\mathrm{ANC}>500 \mu 1^{-1}$ & No \\
\hline Ota et al, 2010 & 2010 & No & 73 & HSCT (auto, allo) & $\begin{array}{l}\text { Itraconazole } 200 \mathrm{mg} \text { per dose } \\
\text { PO/IV OD }\end{array}$ & $\begin{array}{l}400 \text { mg per dose PO/IV } \\
\text { OD }\end{array}$ & $D+28$ & No \\
\hline $\begin{array}{l}\text { Wingard et al, } \\
2010\end{array}$ & 2010 & Yes & 600 & HSCT (allo) & $\begin{array}{l}\text { Voriconazole } 200 \mathrm{mg} \text { per dose } \\
\text { PO BID }\end{array}$ & $400 \mathrm{mg}$ per dose PO OD & $D+100$ & Yes \\
\hline
\end{tabular}

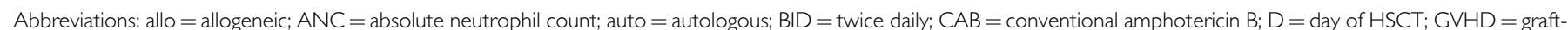
vs-host disease; Hem = haematological; HSCT = haematopoietic stem cell transplantation; IV = intravenous; $N=$ total number of subjects randomised; NS=not specified; $\mathrm{OD}=$ once daily; Pub = publication; $\mathrm{PO}=$ oral; $\mathrm{TID}=$ three times daily. ${ }^{\mathrm{a}}$ Surrogate marker evaluation for invasive fungal infection includes galactomannin and beta- $\mathrm{D}$ glucan testing.

Table 2 Risk of bias assessment of included articles

\begin{tabular}{|c|c|c|c|c|c|c|}
\hline Study author & $\begin{array}{l}\text { Adequate } \\
\text { sequence } \\
\text { generation }\end{array}$ & $\begin{array}{l}\text { Adequate } \\
\text { allocation } \\
\text { concealment }\end{array}$ & Blinding & $\begin{array}{l}\text { Description of } \\
\text { withdrawals } \\
\text { and dropouts }\end{array}$ & $\begin{array}{l}\text { Intention to } \\
\text { treat analysis }\end{array}$ & $\begin{array}{c}\text { Selective } \\
\text { outcome } \\
\text { report }\end{array}$ \\
\hline Bodey et al, 1994 & Yes & Unclear & No & Yes & No & No \\
\hline Annaloro et al, 1995 & Unclear & Unclear & No & No & Yes & No \\
\hline Huijgens et al, 1999a & Unclear & Unclear & Yes & Yes & No & No \\
\hline Morgenstern et al, 1999b & Yes & Inadequate & No & No & No & Yes \\
\hline Timmers et al, $2000 a$ & Unclear & Unclear & No & No & No & No \\
\hline Wolff et al, 2000a & Unclear & Unclear & No & No & Yes & No \\
\hline Koh et al, 2002b & Unclear & Unclear & No & No & Yes & No \\
\hline Winston et al, 2003 & Unclear & Yes & No & Yes & No & No \\
\hline Marr et al, 2004 & Unclear & Unclear & No & Yes & No & No \\
\hline van Burik et al, 2004 & Yes & Yes & Yes & Yes & No & No \\
\hline Choi et al, 2005 & Unclear & Unclear & No & No & No & No \\
\hline Glasmacher et al, 2006 & Yes & Yes & No & Yes & No & No \\
\hline Oren et al, 2006a & Yes & Unclear & No & No & No & No \\
\hline Cornely et al, 2007 & Unclear & Unclear & No & No & Yes & No \\
\hline Ito et al, 2007a & Yes & Unclear & No & Yes & No & No \\
\hline Ullmann et al, 2007 & Unclear & Unclear & Yes & No & Yes & No \\
\hline Hiramatsu et al, 2008a & Unclear & Unclear & No & Yes & No & No \\
\hline Sawada et al, 2009b & Unclear & Yes & No & No & No & No \\
\hline Ota et al, 2010 & Unclear & Unclear & & No & No & No \\
\hline Wingard et al, 2010 & Yes & Yes & Yes & Yes & Yes & No \\
\hline
\end{tabular}


Table 3 Synthesised primary and secondary outcomes of mould-active vs fluconazole prophylaxis

\begin{tabular}{|c|c|c|c|}
\hline Outcome & Trials (patients) & $\operatorname{RR}(95 \% \mathrm{Cl})^{\mathrm{a}}$ & $P$-value \\
\hline Invasive aspergillosis & $15(4503)$ & $0.53(0.37,0.75)$ & 0.0004 \\
\hline Invasive aspergillosis-related mortality & $9(26 \mid 4)$ & $0.62(0.23, \mid .71)$ & 0.36 \\
\hline Overall mortality & $16(4870)$ & $1.00(0.88,1.13)$ & 0.96 \\
\hline
\end{tabular}

Abbreviations: $\mathrm{Cl}=$ confidence interval; $\mathrm{IFI}=$ invasive fungal infection; $\mathrm{RR}=$ risk ratio. ${ }^{\mathrm{a}} \mathrm{RR}<\mathrm{I}$ represents an advantage of mould-active coverage using a random-effects model.

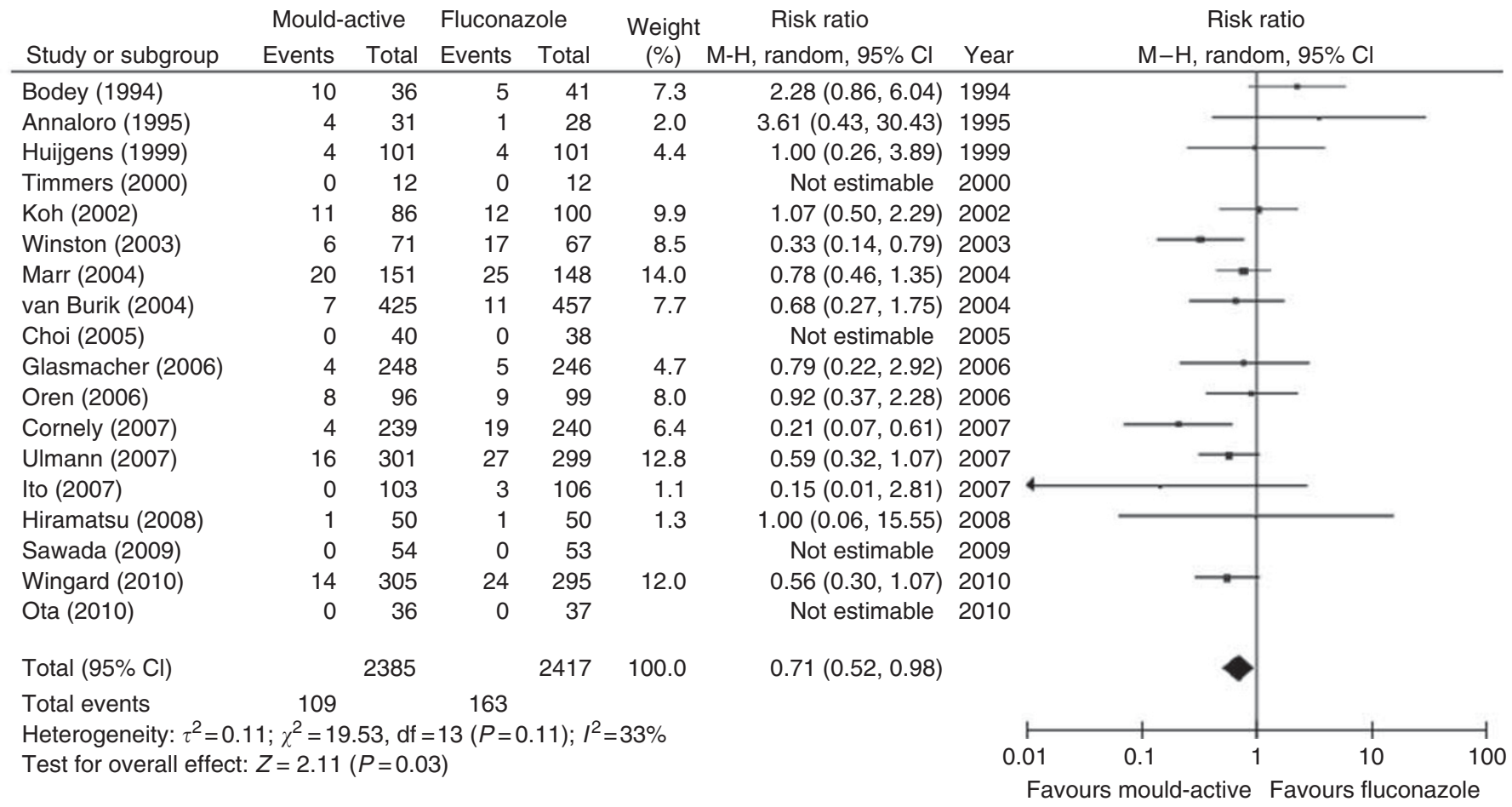

Figure 2 Forest plot of effect of mould-active vs fluconazole prophylaxis on the primary outcome, proven or probable invasive fungal infection. Squares to the left of the vertical line indicate a decreased risk of developing an event in patients receiving mould-active prophylaxis. Horizontal lines through the squares represent $95 \% \mathrm{Cls}$. The diamonds represents the overall RR from the meta-analyses and the corresponding $95 \% \mathrm{Cls}$.

allowed more precise estimation of the effect of mould-active prophylaxis on overall mortality. Furthermore, we examined an additional clinically important outcome, namely adverse events resulting in discontinuation of antifungal prophylaxis, which provides more information to judge the overall utility of mouldactive prophylaxis.

We found that mould-active prophylaxis, when compared with fluconazole prophylaxis, reduces IFI-related mortality but does not influence overall mortality with a point estimate RR of 1.0. The 95\% CI around the overall mortality estimate does not exclude clinically meaningful benefit or harm since the interval was $0.88-1.13$. As IFI-related mortality is a component of overall mortality, it is interesting to see discordance in these two results. There are at least three possibilities to explain this discordance. First, the proportion of IFI-related mortality could be such a small portion of overall mortality that reductions in IFI-related mortality may not detectably impact on mortality. However, there are two observations that argue against this hypothesis. First, the point estimate for overall mortality was 1.0, which suggests no reduction in mortality. Second, mortality was observed for only 3 months and thus, it is hard to envision that IFI-related mortality would be a small proportion of overall mortality within this time frame in these populations. The second possibility that may explain the discrepancy between a reduction in IFD incidence and no effect on overall mortality may relate to the use of galactomannan tests (Marr et al, 2005). Mould-active agents are known to reduce the sensitivity of this test and thus, it is possible that the reduction in IFD seen with anti-mould agents is actually spurious. The third possibility is that mould-active prophylaxis increases non-IFIrelated deaths. This hypothesis is supported by the increase in adverse events observed in the mould-active prophylaxis arm. Furthermore, it is possible that drug interactions further contributed to increased patient deaths.

There are at least three downsides of mould-active antifungal prophylaxis. First, mould-active prophylaxis may be associated with increased adverse events compared with fluconazole prophylaxis as we have demonstrated. Second, mould-active prophylaxis with non-fluconazole azoles may be associated with significant drug interactions and the impact of these interactions has not been fully evaluated. Third is the issue of costs. Many of the mouldactive agents are associated with large costs given the duration of prophylaxis for patients with leukaemia or undergoing allogeneic 


\begin{tabular}{|c|c|c|c|c|}
\hline Outcome & $\begin{array}{c}\text { Trials } \\
\text { (patients) }\end{array}$ & $\begin{array}{c}\mathbf{R R}^{\mathbf{a}} \\
(95 \% \mathrm{Cl})\end{array}$ & $P$-value & $\begin{array}{c}\begin{array}{c}P \text {-value } \\
\text { for }\end{array} \\
\text { interaction } \\
\text { test }\end{array}$ \\
\hline \multicolumn{5}{|l|}{ Mould-active agent } \\
\hline Proven or probable IFI & & & & 0.1 \\
\hline Amphotericin & $3(287)$ & $1.46(0.70,3.05)$ & 0.31 & \\
\hline Other azoles & $12(3426)$ & $0.60(0.43,0.84)$ & 0.003 & \\
\hline Echinocandin & $3(1089)$ & $0.71(0.29,1.73)$ & 0.45 & \\
\hline Invasive aspergillosis & & & & 0.29 \\
\hline Amphotericin & $3(6 \mid 8)$ & $1.18(0.28,4.97)$ & 0.82 & \\
\hline Other azoles & $9(2796)$ & $0.52(0.36,0.76)$ & 0.0006 & \\
\hline Echinocandin & $3(1089)$ & $0.19(0.03,1.11)$ & 0.07 & \\
\hline \multicolumn{5}{|l|}{ IFI-related mortality } \\
\hline Amphotericin & $4(642)$ & $0.91(0.39,2.16)$ & 0.83 & \\
\hline Other azoles & $9(2648)$ & $0.64(0.38,1.08)$ & 0.09 & \\
\hline Echinocandin & $2(982)$ & $0.70(0.12,4.28)$ & 0.70 & \\
\hline \multicolumn{4}{|l|}{ IA-related mortality } & 0.43 \\
\hline Amphotericin & $2(101)$ & $3.41(0.14,81.07)$ & 0.45 & \\
\hline Other azoles & $5(|53|)$ & $0.63(0.18,2.13)$ & 0.46 & \\
\hline Echinocandin & $2(982)$ & $0.27(0.03,2.38)$ & 0.24 & \\
\hline \multicolumn{4}{|l|}{ Overall mortality } & 0.79 \\
\hline Amphotericin & $3(6 \mid 8)$ & I.II $(0.78,1.59)$ & 0.55 & \\
\hline Other azoles & II (3270) & $0.99(0.86,1.14)$ & 0.89 & \\
\hline Echinocandin & $2(982)$ & $0.89(0.42,1.88)$ & 0.76 & \\
\hline \multicolumn{4}{|l|}{$\begin{array}{l}\text { AEs requiring antifungal } \\
\text { treatment discontinuation } \\
\text { or modification }\end{array}$} & 0.001 \\
\hline Amphotericin & $4(642)$ & $5.98(1.20,29.86)$ & 0.03 & \\
\hline Other azoles & $10(2869)$ & $1.92(1.19,3.08)$ & 0.007 & \\
\hline Echinocandin & $2(982)$ & $0.59(0.34,1.03)$ & 0.06 & \\
\hline
\end{tabular}

Abbreviations: $\mathrm{AEs}=$ adverse events; $\mathrm{Cl}=$ confidence interval; $\mid \mathrm{A}=$ invasive aspergillosis; $I F \mid=$ invasive fungal infection; $R R=$ risk ratio. ${ }^{a} R R<\mid$ represents an advantage of mould-active coverage compared with fluconazole using a random-effects model.

HSCT. Cost-effectiveness analyses have shown that posaconazole is a cost-effective strategy for preventing IFI, compared with fluconazole, in patients with GVHD and with acute myeloid leukaemia/myelodysplastic syndrome (Stam et al, 2008; de la Camara et al, 2010; Dranitsaris and Khoury, 2011). In adult patients undergoing HSCT, micafungin has been shown to reduce hospital costs and total patient costs (Schonfeld et al, 2008) and to be cost effective (Sohn et al, 2009), compared with fluconazole. However, these analyses have been based upon single studies rather than synthesised results. Further exploration of costs that take into consideration patient preferences are warranted. Finally, there has been little evaluation of patient preferences for antifungal prophylaxis. Agents such as posaconazole, voriconazole and itraconazole have an oral formulation and thus, may have a lesser impact on quality of life given that administration may occur on an outpatient basis. However, compliance of oral antifungal prophylaxis may be lower (Lehrnbecher et al, 2008). In contrast, amphotericin B formulations and echinocandins are only available in parenteral formulation and, thus, their administration in a prophylactic manner would be expected to have a sizeable impact on quality of life.

This study has several limitations. First and most importantly, we combined several different classes of mould-active antifungals that are expected to have different efficacy and toxicity profiles. However, the stratified analysis failed to illustrate important differences in outcome by mould-active antifungal class. Second, fungal classification and reporting was not consistent in the studies included although we attempted to address this limitation by re-classifying infections using the EORTC/MSG definitions for IFI (Ascioglu et al, 2002). Third, it is possible that surveillance for IFI using galactomannan and beta-D-glucan testing may have altered the efficacy of mould-active prophylaxis. There are an insufficient number of studies that used such testing to be able to explore this effect. Finally, it is also important to mention that only one study included children only, and thus, we are unable to determine if the effect of mould-active prophylaxis compared with fluconazole differs between children and adults. This deficiency supports the need for future randomised trials in children in order determine the effect of mould-active prophylaxis in paediatrics.

Future studies should attempt to better describe the potential benefits and downsides of mould-active prophylaxis. This may be accomplished through future randomised trials of agents thought to be less toxic and through individual patient-level meta-analyses. Furthermore, patient preferences and costs deserve future exploration. Mould-active antifungal prophylaxis may have a large economic impact on care of patients with haematological malignancy and undergoing HSCT; we must be relatively certain of benefits before routine implementation.

In conclusion, this meta-analysis demonstrates that prophylaxis with mould-active compared with fluconazole prophylaxis significantly reduces the number of proven or probable IFI, IA, and IFI-related mortality in patients receiving chemotherapy or undergoing HSCT. However, mould-active antifungal prophylaxis also increases adverse events leading to antifungal modification or discontinuation and does not impact on overall mortality. Future work to better understand the benefits and downsides of individual classes of mould-active antifungals and to explore patient preferences and costs is warranted.

\section{ACKNOWLEDGEMENTS}

We would like to thank Ryoko Ingram who generously assisted with the translation of the article by Sawada into English, as well as Elizabeth Uleryk who provided invaluable feedback for the systematic literature search. LS is supported by a New Investigator Award with the Canadian Institutes of Health Research.

\section{REFERENCES}

Annaloro C, Oriana A, Tagliaferri E, Bertolli V, Della Volpe A, Soligo D, Ibatici A, Pozzoli E, Lambertenghi Deliliers GL (1995) Efficacy of different prophylactic antifungal regimens in bone marrow transplantation. Haematologica 80(6): 512-517

Ascioglu S, Rex JH, de Pauw B, Bennett JE, Bille J, Crokaert F, Denning DW, Donnelly JP, Edwards JE, Erjavec Z, Fiere D, Lortholary O, Maertens J, Meis JF, Patterson TF, Ritter J, Selleslag D, Shah PM, Stevens DA, Walsh TJ. Invasive Fungal Infections Cooperative Group of the European Organization for R, Treatment of CMycoses Study Group of the National Institute of A, Infectious D (2002) Defining opportunistic invasive fungal infections in immunocompromised patients with cancer and hematopoietic stem cell transplants: an international consensus. Clin Infect Diseas 34(1): 7-14

Bodey GP, Anaissie EJ, Elting LS, Estey E, O’Brien S, Kantarjian H (1994) Antifungal prophylaxis during remission induction therapy for acute leukemia fluconazole versus intravenous amphotericin B. Cancer 73(8): 2099-2106

Choi SM, Lee DG, Choi JH, Park SH, Eom KS (2005) Itraconazole oral solution versus fluconazole syrup for prevention of invasive fungal infections in patients receiving hematopoietic stem cell transplantation: prospective, randomized, comparative clinical trial. Infect Chemother 37(2): 71-78

Cornely OA, Bohme A, Buchheidt D, Einsele H, Heinz WJ, Karthaus M, Krause SW, Kruger W, Maschmeyer G, Penack O, Ritter J, Ruhnke M, Sandherr M, Sieniawski M, Vehreschild JJ, Wolf HH, Ullmann AJ. Recommendations of the Infectious Diseases Working Party of the 
German Society for Haematology and Oncology (2009) Primary prophylaxis of invasive fungal infections in patients with hematologic malignancies. Haematologica 94(1): 113-122

Cornely OA, Maertens J, Winston DJ, Perfect J, Ullmann AJ, Walsh TJ, Helfgott D, Holowiecki J, Stockelberg D, Goh Y-T, Petrini M, Hardalo C, Suresh R, Angulo-Gonzalez D (2007) Posaconazole vs fluconazole or itraconazole prophylaxis in patients with neutropenia. $N$ Engl J Med 356(4): 348-359

de la Camara R, Jarque I, Sanz MA, Grau S, Casado MA, Sabater FJ, Carreras E (2010) Economic evaluation of posaconazole vs fluconazole in the prevention of invasive fungal infections in patients with GVHD following haematopoietic SCT. Bone Marrow Transplant 45(5): 925-932

De Pauw B, Walsh TJ, Donnelly JP, Stevens DA, Edwards JE, Calandra T, Pappas PG, Maertens J, Lortholary O, Kauffman CA, Denning DW, Patterson TF, Maschmeyer G, Bille J, Dismukes WE, Herbrecht R Hope WW, Kibbler CC, Kullberg BJ, Marr KA, Munoz P, Odds FC, Perfect JR, Restrepo A, Ruhnke M, Segal BH, Sobel JD, Sorrell TC, Viscoli C, Wingard JR, Zaoutis T, Bennett JE. European Organization for R, Treatment of Cancer/Invasive Fungal Infections Cooperative GNational Institute of AInfectious Diseases Mycoses Study Group Consensus G (2008) Revised definitions of invasive fungal disease from the European Organization for Research and Treatment of Cancer/Invasive Fungal Infections Cooperative Group and the National Institute of Allergy and Infectious Diseases Mycoses Study Group (EORTC/MSG) Consensus Group. Clin Infect Dis 46(12): 1813-1821

Dranitsaris G, Khoury H (2011) Posaconazole versus fluconazole or itraconazole for prevention of invasive fungal infections in patients undergoing intensive cytotoxic therapy for acute myeloid leukemia or myelodysplasia: a cost effectiveness analysis. Support Care Cancer 19(11): $1807-1813$

Ethier MC (2011) Fluconazole Compared to Anti-Mold Coverage for Antifungal Prophylaxis to Prevent Invasive Fungal Infections in Cancer Patients receiving Chemotherapy or after Hematopoietic Stem-Cell Transplantation: A Systematic Review and Meta-Analysis of Randomized Controlled Trials PROSPERO: International prospective register of systematic reviewsCRD420111174. http://www.crd.york.ac.uk/prospero/)

Freifeld AG, Bow EJ, Sepkowitz KA, Boeckh MJ, Ito JI, Mullen CA, Raad, Rolston KV, Young JA, Wingard JR (2011) Clinical practice guideline for the use of antimicrobial agents in neutropenic patients with cancer: 2010 update by the Infectious Diseases Society of America. Clin Infect Dis 52(4): 427-431

Glasmacher A, Cornely O, Ullmann AJ, Wedding U, Bodenstein H, Wandt H, Boewer C, Pasold R, Wolf H-H, Hanel M, Dolken G, Junghanss C, Andreesen R, Bertz H. Itraconazole Research Group of G (2006) An open-label randomized trial comparing itraconazole oral solution with fluconazole oral solution for primary prophylaxis of fungal infections in patients with haematological malignancy and profound neutropenia J Antimicrob Chemother 57(2): 317-325

Glasmacher A, Prentice A, Gorschluter M, Engelhart S, Hahn C, Djulbegovic B, Schmidt-Wolf IG (2003) Itraconazole prevents invasive fungal infections in neutropenic patients treated for hematologic malignancies: evidence from a meta-analysis of 3597 patients. J Clin Oncol 21(24): 4615-4626

Goodman JL, Winston DJ, Greenfield RA, Chandrasekar PH, Fox B Kaizer H, Shadduck RK, Shea TC, Stiff P, Friedman DJ (1992) A controlled trial of fluconazole to prevent fungal infections in patients undergoing bone marrow transplantation. $N$ Engl J Med 326(13): 845-851

Higgins J, Green S (2009) Cochrane Handbook for Systematic Reviews of Interventions Version 5.0.2 (updated September 2009). The Cochrane Collaboration 34(18): 1929-1941

Hiramatsu Y, Maeda Y, Fujii N, Saito T, Nawa Y, Hara M, Yano T, Asakura S, Sunami K, Tabayashi T, Miyata A, Matsuoka K-i, Shinagawa K, Ikeda K, Matsuo K, Tanimoto M, West-Japan H, Oncology G (2008a) Use of micafungin versus fluconazole for antifungal prophylaxis in neutropenic patients receiving hematopoietic stem cell transplantation. Int $J$ Hematol 88(5): 588-595

Hiramatsu Y, Maeda Y, Fujii N, Saito T, Nawa Y, Hara M, Yano T Asakura S, Sunami K, Tabayashi T, Miyata A, Matsuoka K-i, Shinagawa K, Ikeda K, Matsuo K, Tanimoto M, West-Japan H, Oncology G (2008b) Use of micafungin versus fluconazole for antifungal prophylaxis in neutropenic patients receiving hematopoietic stem cell transplantation. Int J Hematol 88(5): 588-595

Huijgens PC, Simoons-Smit AM, van Loenen AC, Prooy E, van Tinteren H, Ossenkoppele GJ, Jonkhoff AR (1999a) Fluconazole versus itraconazole for the prevention of fungal infections in haemato-oncology. J Clin Pathol 52(5): 376-380
Huijgens PC, Simoons-Smit AM, van Loenen AC, Prooy E, van Tinteren H, Ossenkoppele GJ, Jonkhoff AR (1999b) Fluconazole versus itraconazole for the prevention of fungal infections in haemato-oncology. J Clin Pathol 52(5): 376-380

Ito Y, Ohyashiki K, Yoshida I, Takeuchi M, Aoyama Y, Mugitani A, Matsuura Y, Wakita H, Matsuda M, Sakamoto E, Kiguchi T, Urabe A, Tamura K, Kanamaru A, Masaoka T (2007a) The prophylactic effect of itraconazole capsules and fluconazole capsules for systemic fungal infections in patients with acute myeloid leukemia and myelodysplastic syndromes: a Japanese multicenter randomized, controlled study Int J Hematol 85(2): 121-127

Ito Y, Ohyashiki K, Yoshida I, Takeuchi M, Aoyama Y, Mugitani A, Matsuura Y, Wakita H, Matsuda M, Sakamoto E, Kiguchi T, Urabe A, Tamura K, Kanamaru A, Masaoka T (2007b) The prophylactic effect of itraconazole capsules and fluconazole capsules for systemic fungal infections in patients with acute myeloid leukemia and myelodysplastic syndromes: a Japanese multicenter randomized, controlled study Int J Hematol 85(2): 121-127

Koh LP, Kurup A, Goh YT, Fook-Chong SMC, Tan PHC (2002a) Randomized trial of fluconazole versus low-dose amphotericin B in prophylaxis against fungal infections in patients undergoing hematopoietic stem cell transplantation. Am J Hematol 71(4): 260-267

Koh LP, Kurup A, Goh YT, Fook-Chong SMC, Tan PHC (2002b) Randomized trial of fluconazole versus low-dose amphotericin B in prophylaxis against fungal infections in patients undergoing hematopoietic stem cell transplantation. J Clin Pathol 71(4): 260-267

Lehrnbecher T, Ethier MC, Zaoutis T, Creutzig U, Gamis A, Reinhardt D, Aplenc R, Sung L (2009) International variations in infection supportive care practices for paediatric patients with acute myeloid leukaemia Br J Haematol 147(1): 125-128

Lehrnbecher T, Laws HJ, Boehm A, Dworzak M, Janssen G, Simon A, Groll AH (2008) Compliance with anti-infective preventive measures: a multicentre survey among paediatric oncology patients. Eur J Cancer 44(13): 1861-1865

Maertens J, Marchetti O, Herbrecht R, Cornely OA, Fluckiger U, Frere P, Gachot B, Heinz WJ, Lass-Florl C, Ribaud P, Thiebaut A, Cordonnier C. Third European Conference on Infections in L (2011) European guidelines for antifungal management in leukemia and hematopoietic stem cell transplant recipients: summary of the ECIL 3-2009 update. Bone Marrow Transplant 46(5): 709-718

Mahfouz T, Anaissie E (2003) Prevention of fungal infections in the immunocompromised host. Curr Opin Investig Drugs 4(8): 974-990

Marr KA, Crippa F, Leisenring W, Hoyle M, Boeckh M, Balajee SA Nichols WG, Musher B, Corey L (2004) Itraconazole versus fluconazole for prevention of fungal infections in patients receiving allogeneic stem cell transplants. Blood 103(4): 1527-1533

Marr KA, Laverdiere M, Gugel A, Leisenring W (2005) Antifungal therapy decreases sensitivity of the Aspergillus galactomannan enzyme immunoassay. Clin Infect Dis 40(12): 1762-1769

Moher D, Liberati A, Tetzlaff J, Altman DG, Group P (2009) Preferred reporting items for systematic reviews and meta-analyses: the PRISMA statement. Ann Int Med 151(4): 264-269

Morgenstern GR, Prentice AG, Prentice HG, Ropner JE, Schey SA, Warnock DW. U.K. Multicentre Antifungal Prophylaxis Study Group (1999a) A randomized controlled trial of itraconazole versus fluconazole for the prevention of fungal infections in patients with haematological malignancies. Br J Haematol 105(4): 901-911

Morgenstern GR, Prentice AG, Prentice HG, Ropner JE, Schey SA, Warnock DW. U.K. Multicentre Antifungal Prophylaxis Study Group (1999b) A randomized controlled trial of itraconazole versus fluconazole for the prevention of fungal infections in patients with haematological malignancies. Br J Haematol 105(4): 901-911

Oren I, Rowe JM, Sprecher H, Tamir A, Benyamini N, Akria L, Gorelik A, Dally N, Zuckerman T, Haddad N, Fineman R, Dann EJ (2006a) A prospective randomized trial of itraconazole vs fluconazole for the prevention of fungal infections in patients with acute leukemia and hematopoietic stem cell transplant recipients. Bone Marrow Transplant 38(2): 127-134

Oren I, Rowe JM, Sprecher H, Tamir A, Benyamini N, Akria L, Gorelik A, Dally N, Zuckerman T, Haddad N, Fineman R, Dann EJ (2006b) A prospective randomized trial of itraconazole vs fluconazole for the prevention of fungal infections in patients with acute leukemia and hematopoietic stem cell transplant recipients. Bone Marrow Transplant 38(2): $127-134$ 
Ota S, Matsukawa T, Kosugi M, Shima K, Katsura Y, Kanaya M, Matsuoka S, Noguchi S, Fujii S, Nakata M, Imai K, Hirano T, Kobayashi N, Ogasawara M, Kiyama Y, Kasai M (2010) Itraconazole versus fluconazole for prophylaxis against invasive fungal infections during neutropenia in patients receiving haematopoietic stem cell transplants. Bone Marrow Transplant. Conference: 36th Annual Meeting of the European Group for Blood and Marrow Transplantation, EBMT 2010 Vienna Austria. Conference Start: 20100321 Conference End: 20100324. Conference Publication: (var.pagings) 45: S394

Pagano L, Caira M, Candoni A, Offidani M, Martino B, Specchia G, Pastore D, Stanzani M, Cattaneo C, Fanci R, Caramatti C, Rossini F, Luppi M, Potenza L, Ferrara F, Mitra ME, Fadda RM, Invernizzi R, Aloisi T, Picardi M, Bonini A, Vacca A, Chierichini A, Melillo L, de Waure C, Fianchi L, Riva M, Leone G, Aversa F, Nosari A (2010) Invasive aspergillosis in patients with acute myeloid leukemia: a SEIFEM-2008 registry study. Haematologica 95(4): 644-650

Prentice AG, Glasmacher A, Djulbegovic B (2006) In meta-analysis itraconazole is superior to fluconazole for prophylaxis of systemic fungal infection in the treatment of haematological malignancy. $\mathrm{Br} J$ Haematol 132(5): 656-658

Robenshtok E, Gafter-Gvili A, Goldberg E, Weinberger M, Yeshurun M, Leibovici L, Paul M (2007) Antifungal prophylaxis in cancer patients after chemotherapy or hematopoietic stem-cell transplantation: systematic review and meta-analysis. J Clin Oncol 25(34): 5471-5489

Rotstein C, Bow EJ, Laverdiere M, Ioannou S, Carr D, Moghaddam N. The Canadian Fluconazole Prophylaxis Study Group (1999) Randomized placebo-controlled trial of fluconazole prophylaxis for neutropenic cancer patients: benefit based on purpose and intensity of cytotoxic therapy. Clin Infect Dis 28(2): 331-340

Sawada A, Sakata N, Higuchi B, Takeshita Y, Ishihara T, Sakata A, Kouroki M, Kondo O, Koyama M, Hirano S, Yasui M, Inoue M, Yoshioka A, Kawa $\mathrm{K}$ (2009a) Comparison of micafungin and fosfluconazole as prophylaxis for invasive fungal infection during neutropenia in children undergoing chemotherapy and hematopoietic stem cell transplantation. Rinsho Ketsueki-Japanese J Clin Hematol 50(12): 1692-1699

Sawada A, Sakata N, Higuchi B, Takeshita Y, Ishihara T, Sakata A, Kouroki M, Kondo O, Koyama M, Hirano S, Yasui M, Inoue M, Yoshioka A, Kawa $\mathrm{K}$ (2009b) Comparison of micafungin and fosfluconazole as prophylaxis for invasive fungal infection during neutropenia in children undergoing chemotherapy and hematopoietic stem cell transplantation. Rinsho Ketsueki 50(12): 1692-1699

Schonfeld W, Wang Cheng J, Tong KB, Seifeldin R (2008) Cost-effectiveness analysis of antifungal prophylaxis in patients undergoing hematopoietic stem cell transplantation. Clin Ther 30(5): 964-973

Slavin MA, Osborne B, Adams R, Levenstein MJ, Schoch HG, Feldman AR, Meyers JD, Bowden RA (1995) Efficacy and safety of fluconazole prophylaxis for fungal infections after marrow transplantationa prospective, randomized, double-blind study. J Infect Dis 171(6): $1545-1552$

Sohn HS, Lee TJ, Kim J, Kim D (2009) Cost-effectiveness analysis of micafungin versus fluconazole for prophylaxis of invasive fungal infections in patients undergoing hematopoietic stem cell transplantation in Korea. Clin Ther 31(5): 1105-1115, discussion 1066-1068

Stam WB, O'Sullivan AK, Rijnders B, Lugtenburg E, Span LF, Janssen JJ, Jansen JP (2008) Economic evaluation of posaconazole $v s$ standard azole prophylaxis in high risk neutropenic patients in the Netherlands. Eur J Haematol 81(6): 467-474

Sutton AJ, Duval SJ, Tweedie RL, Abrams KR, Jones DR (2000) Empirical assessment of effect of publication bias on meta-analyses. BMJ 320(7249): 1574-1577

Timmers GJ, Zweegman S, Simoons-Smit AM, van Loenen AC, Touw D, Huijgens PC (2000a) Amphotericin B colloidal dispersion (Amphocil) vs fluconazole for the prevention of fungal infections in neutropenic patients: data of a prematurely stopped clinical trial. Bone Marrow Transplant 25(8): 879-884

Timmers GJ, Zweegman S, Simoons-Smit AM, van Loenen AC, Touw D, Huijgens PC (2000b) Amphotericin B colloidal dispersion (Amphocil) vs fluconazole for the prevention of fungal infections in neutropenic patients: data of a prematurely stopped clinical trial. Bone Marrow Transplantation 25(8): 879-884

Ullmann AJ, Lipton JH, Vesole DH, Chandrasekar P, Langston A, Tarantolo SR, Greinix H, Morais de Azevedo W, Reddy V, Boparai N, Pedicone L, Patino H, Durrant S (2007) Posaconazole or fluconazole for prophylaxis in severe graft-versus-host disease. [Erratum appears in N Engl J Med. 2007 Jul 26;357(4):428] N Engl J Med 356(4): 335-347

van Burik J-AH, Ratanatharathorn V, Stepan DE, Miller CB, Lipton JH, Vesole DH, Bunin N, Wall DA, Hiemenz JW, Satoi Y, Lee JM, Walsh TJ. National Institute of AInfectious Diseases Mycoses Study G (2004) Micafungin versus fluconazole for prophylaxis against invasive fungal infections during neutropenia in patients undergoing hematopoietic stem cell transplantation. Clin Infect Dis 39(10): 1407-1416

Walsh TJ, Anaissie EJ, Denning DW, Herbrecht R, Kontoyiannis DP, Marr KA, Morrison VA, Segal BH, Steinbach WJ, Stevens DA, van Burik JA, Wingard JR, Patterson TF. Infectious Diseases Society of A (2008) Treatment of aspergillosis: clinical practice guidelines of the Infectious Diseases Society of America. Clin Infect Dis 46(3): 327-360

Wingard JR, Carter SL, Walsh TJ, Kurtzberg J, Small TN, Baden LR, Gersten ID, Mendizabal AM, Leather HL, Confer DL, Maziarz RT, Stadtmauer EA, Bolanos-Meade J, Brown J, Dipersio JF, Boeckh M, Marr KA. Blood, Marrow Transplant Clinical Trials N (2010) Randomized, double-blind trial of fluconazole versus voriconazole for prevention of invasive fungal infection after allogeneic hematopoietic cell transplantation. Blood 116(24): 5111-5118

Winston DJ, Maziarz RT, Chandrasekar PH, Lazarus HM, Goldman M, Blumer JL, Leitz GJ, Territo MC (2003) Intravenous and oral itraconazole versus intravenous and oral fluconazole for long-term antifungal prophylaxis in allogeneic hematopoietic stem-cell transplant recipients. A multicenter, randomized trial. Ann Int Med 138(9): 705-713

Wolff SN, Fay J, Stevens D, Herzig RH, Pohlman B, Bolwell B, Lynch J, Ericson S, Freytes CO, LeMaistre F, Collins R, Pineiro L, Greer J, Stein R, Goodman SA, Dummer S (2000a) Fluconazole vs low-dose amphotericin $\mathrm{B}$ for the prevention of fungal infections in patients undergoing bone marrow transplantation: a study of the North American Marrow Transplant Group. Bone Marrow Transplant 25(8): 853-859

Wolff SN, Fay J, Stevens D, Herzig RH, Pohlman B, Bolwell B, Lynch J, Ericson S, Freytes CO, LeMaistre F, Collins R, Pineiro L, Greer J, Stein R, Goodman SA, Dummer S (2000b) Fluconazole vs low-dose amphotericin $\mathrm{B}$ for the prevention of fungal infections in patients undergoing bone marrow transplantation: a study of the North American Marrow Transplant Group. Bone Marrow Transplant 25(8): 853-859 
APPENDIX

Table Al Search strategies used to identify randomised study of mould-active vs fluconazole antifungal prophylaxis in patients with cancer or undergoing haematopoietic stem cell transplantation

Ovid MEDLINE(R) 1948 to August (week 2) 2011 (run on August 24, 201 I)

I Fluconazole/ or (fluconazol* or flucolich or arnazole or beagyne or elazor or flucobeta or solacap or diflucan or triflucan or

'uk 49858' or uk49858 or neofomiral or lavisa or zonal or 'fluc hexal' or fluchexal or oxifungol or fungata or loitin or flunazul or zoltrix).mp.

2 exp Aspergillus/pc or (exp Aspergillus/ and (prophyla* or prevent*).mp.) or exp Mycoses/pc or (exp Mycoses/ and (prophyla* or prevent*).mp.) or (prophylaxis or (prevent* adj2 (fungal or fungus))).ti,ab.

3 Stem Cell Transplantation.mp. or exp Stem Cell Transplantation/

4 exp neoplasms/ or (cancer or oncolog*).mp.

exp Neutropenia/ or neutropeni*.mp.

3 or 4 or 5

1 and 2 and 6

randomised controlled trial.pt.

controlled clinical trial.pt.

10 randomised.ab.

II drug therapy.fs.

12 randomly.ab.

13 trial.ab.

14 groups.ab.

158 or 9 or 10 or 11 or 12 or 13 or 14

16 exp animals/ not humans.sh.

$17 \quad 15$ not 16

$18 \quad 7$ and 17

Database: EMBASE < 1980 to 2011 Week 33> (run on 24 August 2011)

I fluconazole/ or (fluconazol* or flucolich or arnazole or beagyne or elazor or flucobeta or solacap or diflucan or triflucan or 'uk 49858 ' or uk49858 or neofomiral or lavisa or zonal or 'fluc hexal' or fluchexal or oxifungol or fungata or loitin or flunazul or zoltrix or Afungil or Alflucoz or Baten or Biocanol or Biozolene or CCRIS 72II or Canzol or Cryptal or DRG-0005 or Dimycon or Elazor or Mutum or Pritenzol or Syscan or Triconal or Zemyc or Zoltec).mp.

2 (exp Aspergillus/ and (prevent* or prophyla*)).mp. or exp mycosis/pc or (exp mycosis/ and (prevent* or prophyla*)).mp. or ((exp Aspergillus/ or exp mycosis/) and (prophylaxis/ or infection prevention/))

3 stem cell transplantation.mp. or exp stem cell transplantation/

exp neoplasms/ or (cancer or oncolog*).mp.

exp NEUTROPENIA/ or exp FEBRILE NEUTROPENIA/ or neutropenia.mp.

3 or 4

1 and 2 and 6

randomised controlled trial/ or ct.fs. or random\$.mp. or doubl\$adj blind\$.mp.

7 and 8

EBM Reviews-Cochrane Central Register of Controlled Trials, 3rd Quarter 2011 (run on 24 August 2011)

I fluconazole/ or (fluconazol* or flucolich or arnazole or beagyne or elazor or flucobeta or solacap or diflucan or triflucan or 'uk 49858' or uk49858 or neofomiral or lavisa or zonal or 'fluc hexal' or fluchexal or oxifungol or fungata or loitin or flunazul or zoltrix or Afungil or Alflucoz or Baten or Biocanol or Biozolene or CCRIS 72I I or Canzol or Cryptal or DRG-0005 or Dimycon or Elazor or Mutum or Pritenzol or Syscan or Triconal or Zemyc or Zoltec).mp.

2 exp Aspergillus/pc or (exp Aspergillus/ and (prophyla* or prevent*).mp.) or exp Mycoses/pc or (exp Mycoses/ and (prophyla* or prevent*).mp.) or (prophylaxis or (prevent* adj2 (fungal or fungus))).ti,ab.

3 Stem Cell Transplantation.mp. or exp Stem Cell Transplantation/ or exp Bone Marrow Transplantation/ 2679

4 exp neoplasms/ or (cancer or oncolog*) mp.

neutropenia.mp. or exp Neutropenia/

Table A2 Stratified analyses by blinding and intention to treat analysis

\begin{tabular}{|c|c|c|c|c|}
\hline Outcome & Trials (patients) & $\mathbf{R R}^{*}(95 \% \mathrm{Cl})$ & $P$-value & $P$-value for interaction test \\
\hline \multicolumn{5}{|l|}{ Blinding } \\
\hline Proven or probable IFI & & & & 0.52 \\
\hline Blinded & $4(2284)$ & $0.62(0.42,0.91)$ & 0.01 & \\
\hline Not blinded & $14(25 \mid 8)$ & $0.71(0.52,0.98)$ & 0.27 & \\
\hline Invasive aspergillosis & & & & 0.60 \\
\hline Blinded & $4(2284)$ & $0.47(0.23,0.98)$ & 0.04 & \\
\hline Not blinded & $11(22196)$ & $0.59(0.37,0.95)$ & 0.03 & \\
\hline
\end{tabular}


Table A2 (Continued)

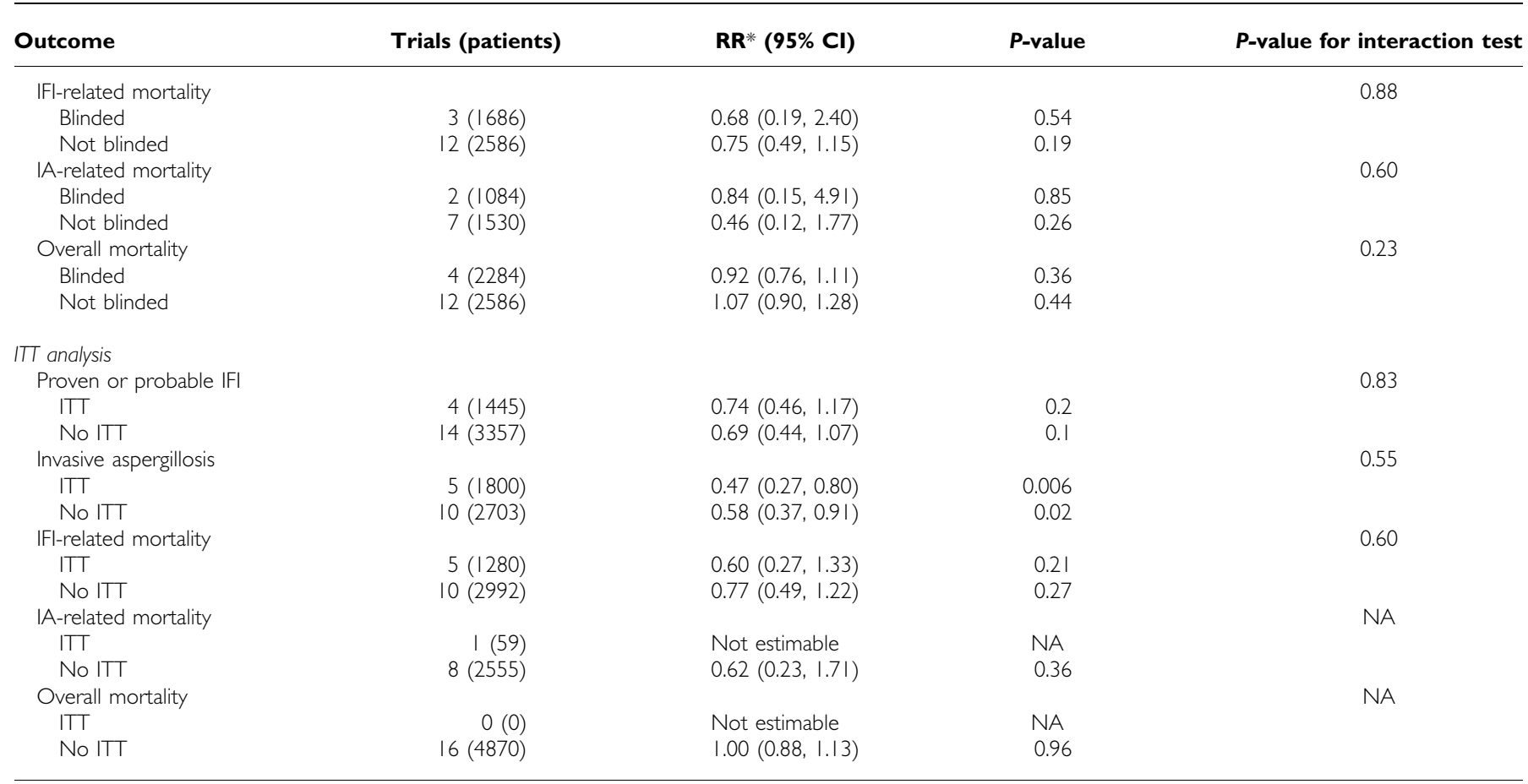

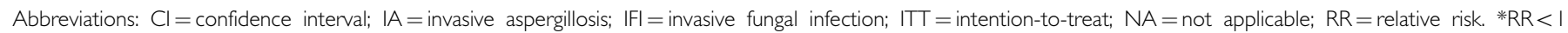
represents an advantage of mould-active coverage compared with fluconazole using a random-effects model.

Table A3 Stratified analyses by study population and fluconazole dose

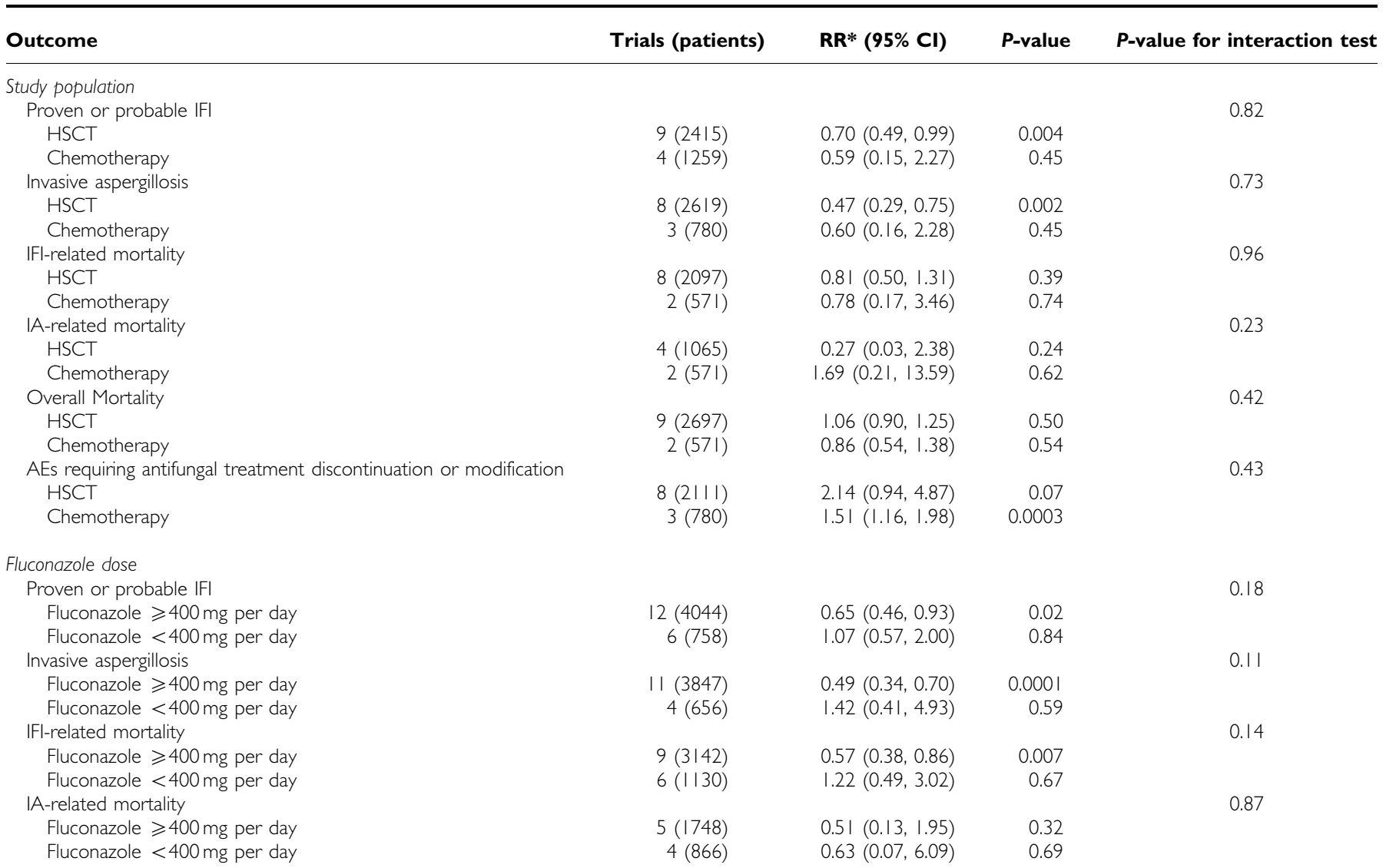


Table A3 (Continued)

\section{Outcome}

Overall mortality

Fluconazole $\geqslant 400 \mathrm{mg}$ per day

Fluconazole $<400 \mathrm{mg}$ per day

AEs requiring antifungal treatment discontinuation or modification

Fluconazole $\geqslant 400 \mathrm{mg}$ per day

Fluconazole $<400 \mathrm{mg}$ per day

\section{Trials (patients)}

$\mathbf{R R}^{*}(95 \% \mathrm{CI})$

P-value

$P$-value for interaction test

$10(3740)$
$6(1130)$
$10(3213)$
$6(1280)$

0.76

0.92

$1.03(0.57,1.88)$

0.12

$1.49(0.91,2.43)$

0.12

$3.19(2.01,5.05)$

0.0001

0.87
Abbreviations: $\mathrm{AEs}=$ adverse events; $\mathrm{Cl}=$ confidence interval; $\mathrm{HSCT}=$ haematopoietic stem cell transplantation; $\mid \mathrm{A}=$ invasive aspergillosis; $\mathrm{IFI}=$ invasive fungal infection; $\mathrm{ITT}=$ intention-to-treat; $\mathrm{RR}=$ relative risk. $* \mathrm{RR}<\mathrm{I}$ represents an advantage of mould-active coverage compared with fluconazole using a random-effects model.

Table A4 Meta-regression for primary and secondary outcomes of mould-active vs fluconazole prophylaxis

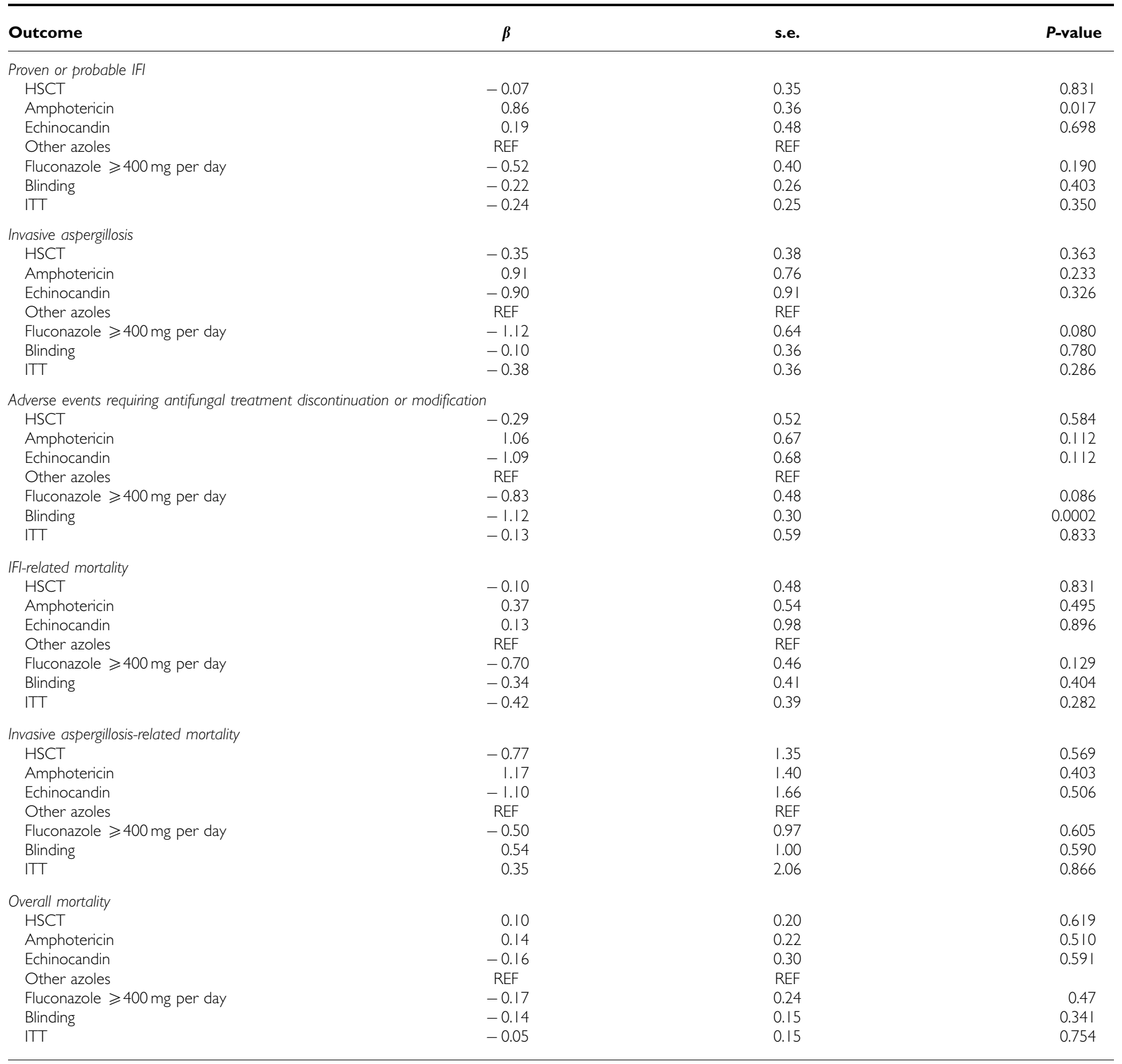

Abbreviations: HSCT = haematopoietic stem cell transplantation; IFI = invasive fungal infection; ITT = intention-to-treat; REF = reference category; s.e. = standard error. 
Table A5 Sensitivity analyses for primary and secondary outcomes of mould-active vs fluconazole prophylaxis

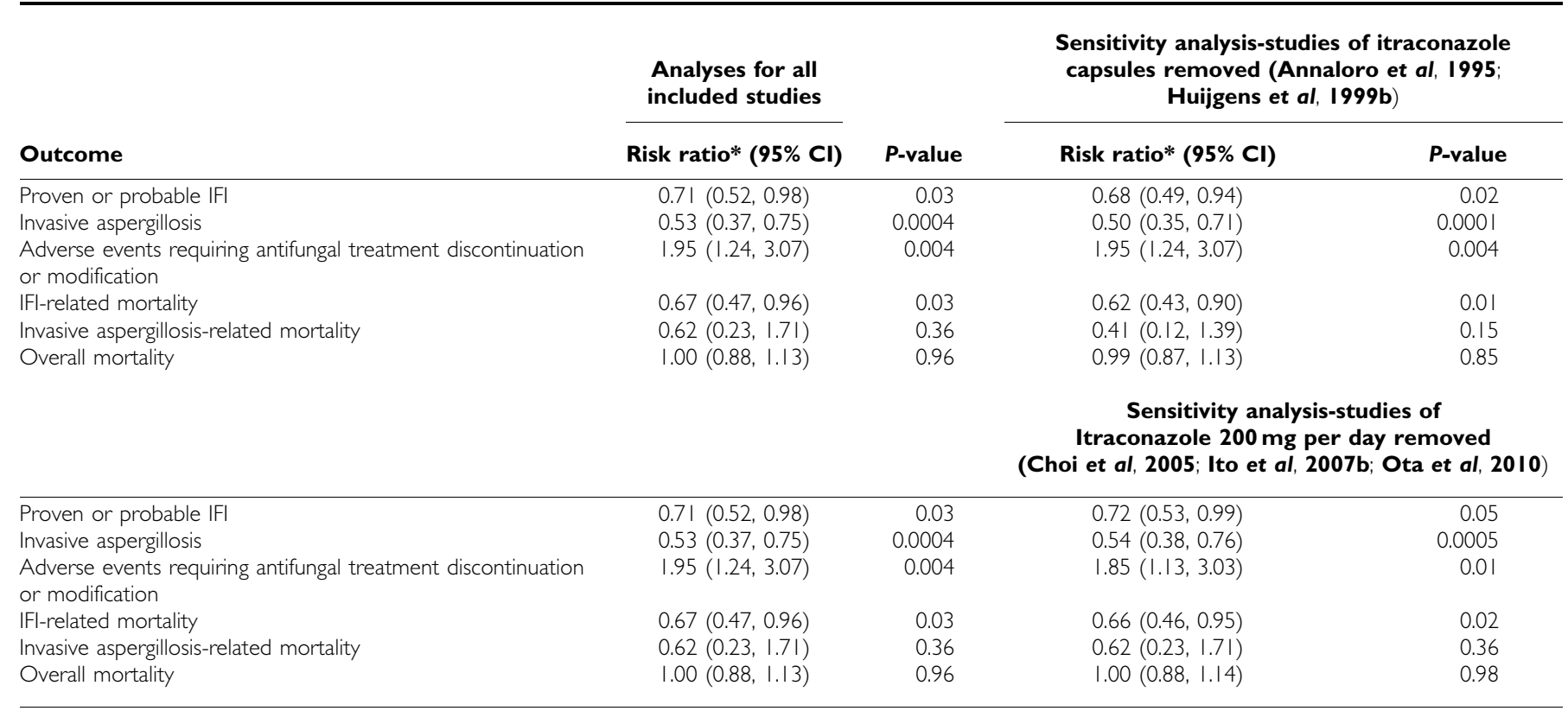

Abbreviations: $\mathrm{Cl}$ = confidence interval; $\mathrm{IFI}=$ invasive fungal infection. $* \mathrm{RR}<\mid$ represents an advantage of mould-active coverage compared with fluconazole using a randomeffects model.

This work is published under the standard license to publish agreement. After 12 months the work will become freely available and the license terms will switch to a Creative Commons Attribution-NonCommercial-Share Alike 3.0 Unported License. 